\title{
Cellular Distribution of a Gold(I) Based Anti-Cancer Compound: a Complementary NanoSIMS and EFTEM Study
}

\author{
L.E. Wedlock, ${ }^{*}$ S. J. Berners-Price, ${ }^{*}$ J.B. Cliff, ${ }^{* *}$ L. Filgueira, ${ }^{* * *}$ M.R. Kilburn, ${ }^{* *}$ and M. \\ Saunders** \\ * Discipline of Chemistry, School of Biomedical, Biomolecular and Chemical Sciences, The \\ University of Western Australia, Crawley WA, 6009, Australia \\ ** Centre for Microscopy, Characterization and Analysis, The University of Western Australia, \\ Crawley WA, 6009, Australia \\ *** School of Anatomy and Human Biology, The University of Western Australia, Crawley WA, \\ 6009, Australia
}

The development of successful chemotherapeutics faces two main problems: acquired resistance to drugs and lack of selectivity of the drugs for tumor cells over normal cells. This has resulted in increasing interest in targeted drug design; for example, design of drugs that selectively target the mitochondria of tumour cells because of their pivotal role in apoptosis. Au(I) lipophilic cations are promising chemotherapeutic candidates in this regard, with growing evidence for their antimitochondrial mechanism of action $[1,2]$. However, a deeper understanding of their function and sub-cellular localisation is needed. The availability of suitable imaging techniques to map the subcellular distribution of the drugs is therefore essential.

Nano-scale Secondary Ion Mass Spectrometry (NanoSIMS) and energy-filtered transmission electron microscopy (EFTEM) are two techniques that potentially have the ability to track the uptake and distribution of these $\mathrm{Au}$ containing drugs. We have applied these techniques in tandem to investigate the sub-cellular localization of a known $\mathrm{Au}(\mathrm{I})$ phosphine compound, [Au(d2pype)2]Cl (where d2pype is 1,2-bis(di-2-pyridylphosphino)ethane), inside human breast adenocarcinoma (MDA) cells. Both NanoSIMS and EFTEM are imaging techniques that allow for the direct mapping of elements whilst maintaining the ability to observe the morphological features of the cell. This offers a distinct advantage over ICP-MS, which has been used for determining cellular uptake of $\mathrm{Au}(\mathrm{I})$ compounds at the bulk-cell-culture scale and can only be applied to subcellular components by using physical separation techniques.

Secondary ion maps of ${ }^{197} \mathrm{Au},{ }^{12} \mathrm{C}^{14} \mathrm{~N}$ and ${ }^{31} \mathrm{P}$ were acquired from resin-embedded sections of MDA cells exposed to the compound for various times, using a Cameca NanoSIMS 50. The ${ }^{12} \mathrm{C}^{14} \mathrm{~N}$ and ${ }^{31} \mathrm{P}$ signals are emitted with varying intensities from different cell components and this contrast allows the direct visualization of the cell morphology, whilst the ${ }^{197} \mathrm{Au}$ signal originates directly from the compound. High-resolution NanoSIMS images clearly show the distribution of Au within cells after incubation (Fig. 1). This demonstrates the power of this technique for imaging drug targeting at the sub-cellular scale. EFTEM element maps for Au were obtained from similar sections using the traditional three-window method for background subtraction. The $\mathrm{Au} \mathrm{O}_{2,3}$ edge at $\sim 54 \mathrm{eV}$ was used, with optimization of the pre and post-edge window energies to obtain suitable background removal (Fig. 2). For shorter incubation times, the Au concentration was below the detectable limit for EFTEM but was detectable with NanoSIMS. For longer incubations, EFTEM data were comparable with NanoSIMS data. The ability of TEM to image the ultrastructure of the cells provided additional insight into the mechanism of action and ultrastructural modifications associated with uptake of the compound. The different sensitivities and resolutions of NanoSIMS and TEM are highly 
complementary, allowing both direct mapping of the Au compound at the sub-cellular level and clear identification of the cell ultrastructure simultaneously.

\section{References}

[1] P. J. Barnard, S. J. Berners-Price, Coord. Chem. Rev. 251 (2007) 1889.

[2] S.J. Berners-Price, A. Filipovska, Aust. J. Chem. 61 (2008) 661.

[3] All microscopy analyses were carried out using facilities at the Centre for Microscopy, Characterization and Analysis at the University of Western Australia, which is supported by University, State and Federal funding. This project was supported by a Graduate Research Student Training Grant to LEW (from UWA), an AMMRF TAP Grant to LEW and ARC Discovery Project DP0986318. We thank Coby Sutcliffe for the synthesis of [Au(d2pype $\left.)_{2}\right]$.

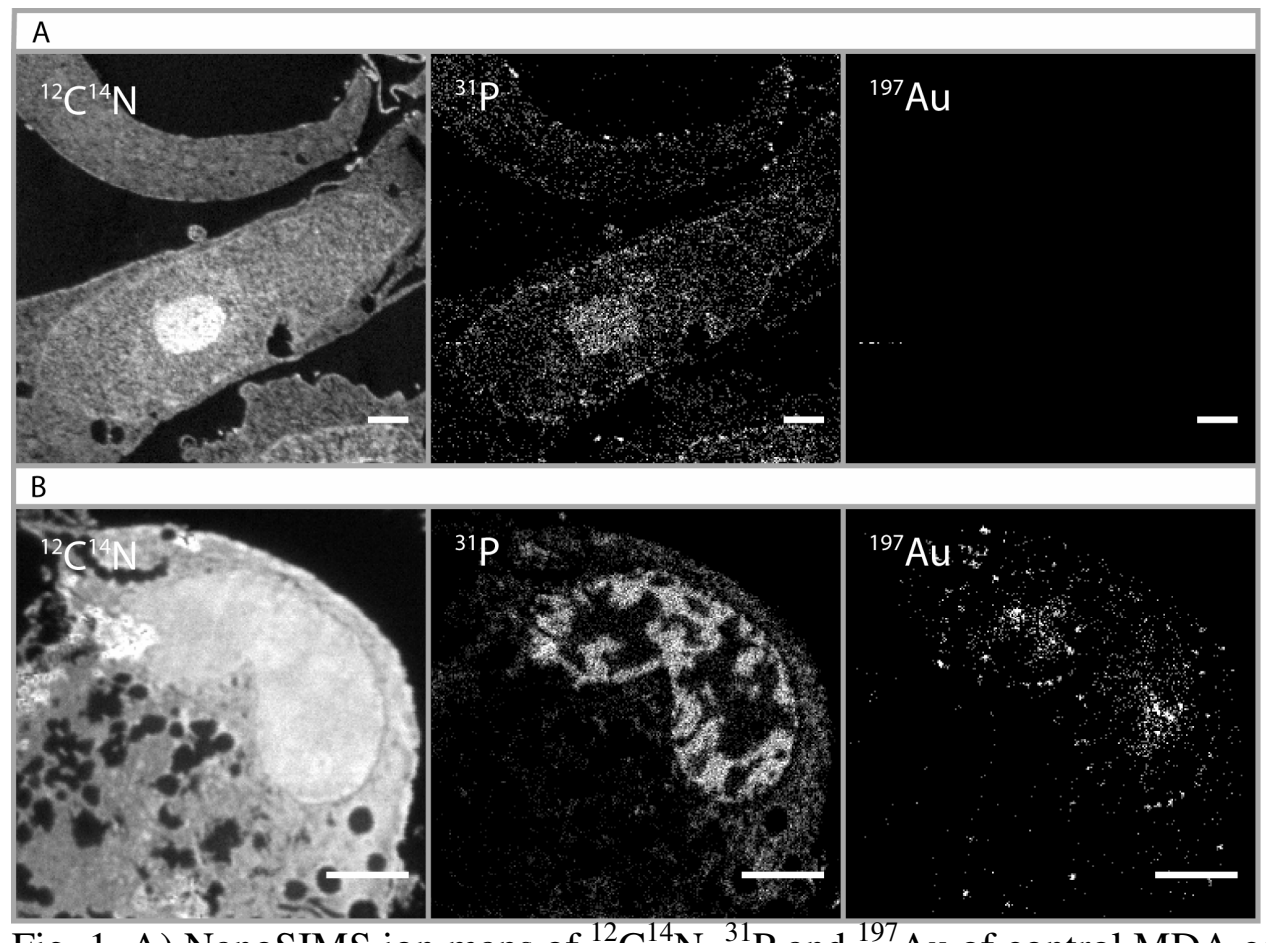

Fig. 1. A) NanoSIMS ion maps of ${ }^{12} \mathrm{C}^{14} \mathrm{~N},{ }^{31} \mathrm{P}$ and ${ }^{197} \mathrm{Au}$ of control MDA cells; B) Ion maps of MDA cells after $2 \mathrm{~h}$ treatment with [Au(d2pype $)_{2}$ ] Ion maps demonstrate that after $2 \mathrm{~h}$ treatment, $\mathrm{Au}$ is localized in the nucleus but is antithetic to P. Scale bars $=2 \mu \mathrm{m}$.

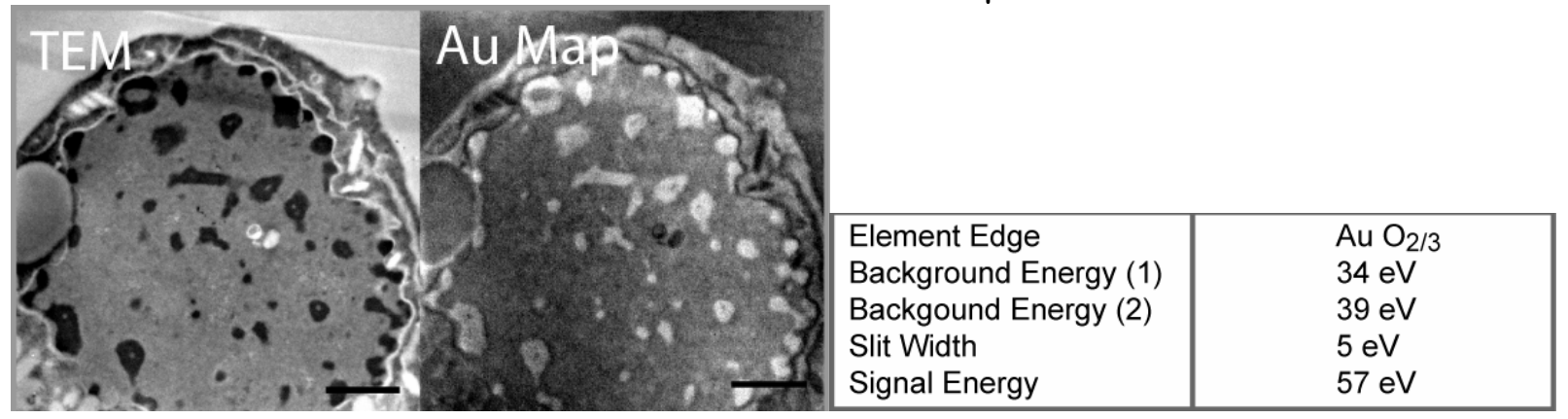

Fig. 2. Conventional TEM and corresponding EFTEM Au map of a nuclear region of an MDA cell after $4 \mathrm{~h}$ treatment with the compound. Aggregates of electron dense material appear as bright spots in the Au map. Scale bars $=2 \mu \mathrm{m}$. Optimized parameters for mapping the Au edge are also shown. 